\title{
3 Research Square

\section{Human papillomavirus type 68 prevalence and genetic variability based on E6/E7 genes in Sichuan}

Jiaoyu He

Sichuan University

Qiufu Li

Sichuan University

Yuning Chen

Xindu District people's Hospital of chengdu

Jianying Peng

Sichuan University

Shiyu Ma

Sichuan University

Zhilin Song

Sichuan University

Yiran Liu

Sichuan University

Yanru Cui

Sichuan University

Junhang Deng

Sichuan University

Xia Wei

Sichuan University

Xianping Ding ( $\nabla$ brainding@scu.edu.cn )

Sichuan University https://orcid.org/0000-0003-1555-5028

\section{Research}

Keywords: HPV68, genetic variability, phylogeny, selection pressure, epitopes

Posted Date: March 23rd, 2021

DOI: https://doi.org/10.21203/rs.3.rs-310542/v1

License: (c) (1) This work is licensed under a Creative Commons Attribution 4.0 International License.

Read Full License 
Version of Record: A version of this preprint was published at Virology on December 1st, 2021. See the published version at https://doi.org/10.1016/j.virol.2021.11.015. 


\section{Abstract}

Background:

Cervical cancer is one of the malignant tumors threatening women's health worldwide, only second to breast cancer. $99.7 \%$ cervical cancer was found to be associated with high-risk HPV (HR-HPV) persistent infections. HPV68 is a common HR-HPV, closely related to cervical cancer.

Methods:

Cell samples were collected by cervical scraped for HPV detecting and typing, and HPV 68 positive samples were selected out. Important E6, E7 genes of HPV 68 were sequenced and analyzed for the study of HPV 68 genetic polymorphisms. Phylogenetic tree of E6-E7 was constructed by Maximum likelihood method of MEGA v7.0 software. The selection pressure sites of HPV68 E6, E7 were predicted by codeml in the PAML 4.8. The secondary structure and three-dimensional structure of HPV68 E6, E7 were analyzed by PSIPRED server and SWISS-MODEL respectively. T-cell and B-cell antigen epitopes of HPV68 E6, E7 were predicted by immune epitope Database Analysis (IEDB) resource and ABCpred server respectively

Results:

a total of 10650 cell samples were collected for detecting and typing, and $2939(27.60 \%, 2939 / 10650)$ positive samples were detected, 174 (5.92\%, 174/2939) were HPV68; 150 HPV68 E6-E7 were successful amplified and analyzed, 32 nucleotide mutations were observed in this study, 50\% (16/32) were nonsynonymous mutations; among them, 4 non-synonymous mutations were detected in E6 gene (one in the Coil and three in the Strand), 12 were in E7 gene (four in the alpha helix, two in the Coil and six in the Strand). Phylogenetic analysis of HPV68 E6-E7 suggested that $C$ was the most frequent HPV68 lineage in Sichuan China. 82-90SESVYATTL, 85-93VYATTLETI, 13-21KLPDLCRTL and 67-81-SCIKFYAKIRELRYY, 6882CIKFYAKIRELRYYS, 66-80QSCIKFYAKIRELRY were the most potential HPV68 E6 HLA-囚, HLA-】epitopes respectively; and B-cell epitopes were 138-153CRHCWTSKREDRRRTR, 82-97SESVYATTLETITNTK. 92101LLFMDSLNFV, 45-53AVNHHQHQL and 20-35EIEPVDLVCHEQLGDS were the most potential HPV68 E7 HLA- $\$ and B-cell epitopes. 3 HPV68 E6 positive selection sites and 5 HPV68 E7 were detected, and they all had a certain influence on the proteins structure and epitopes affinity.

Conclusion:

Non-synonymous mutations of HPV 68 located in positive selection sites resulted in differences in protein structure and epitopes affinity, that may affect the pathogenicity and adaptability of HPV68 to the environment. HPV68 data enrichment is of great significance for understanding the inherent geographical and biological differences of HPV68 in china; and targeting potential epitopes for therapeutic vaccines may improve the effective of vaccines design for specific populations.

\section{Full Text}


Due to technical limitations, full-text HTML conversion of this manuscript could not be completed. However, the latest manuscript can be downloaded and accessed as a PDF.

\section{Tables}

Due to technical limitations, Tables 1 and 2 are only available as a download in the supplemental files section

Figures

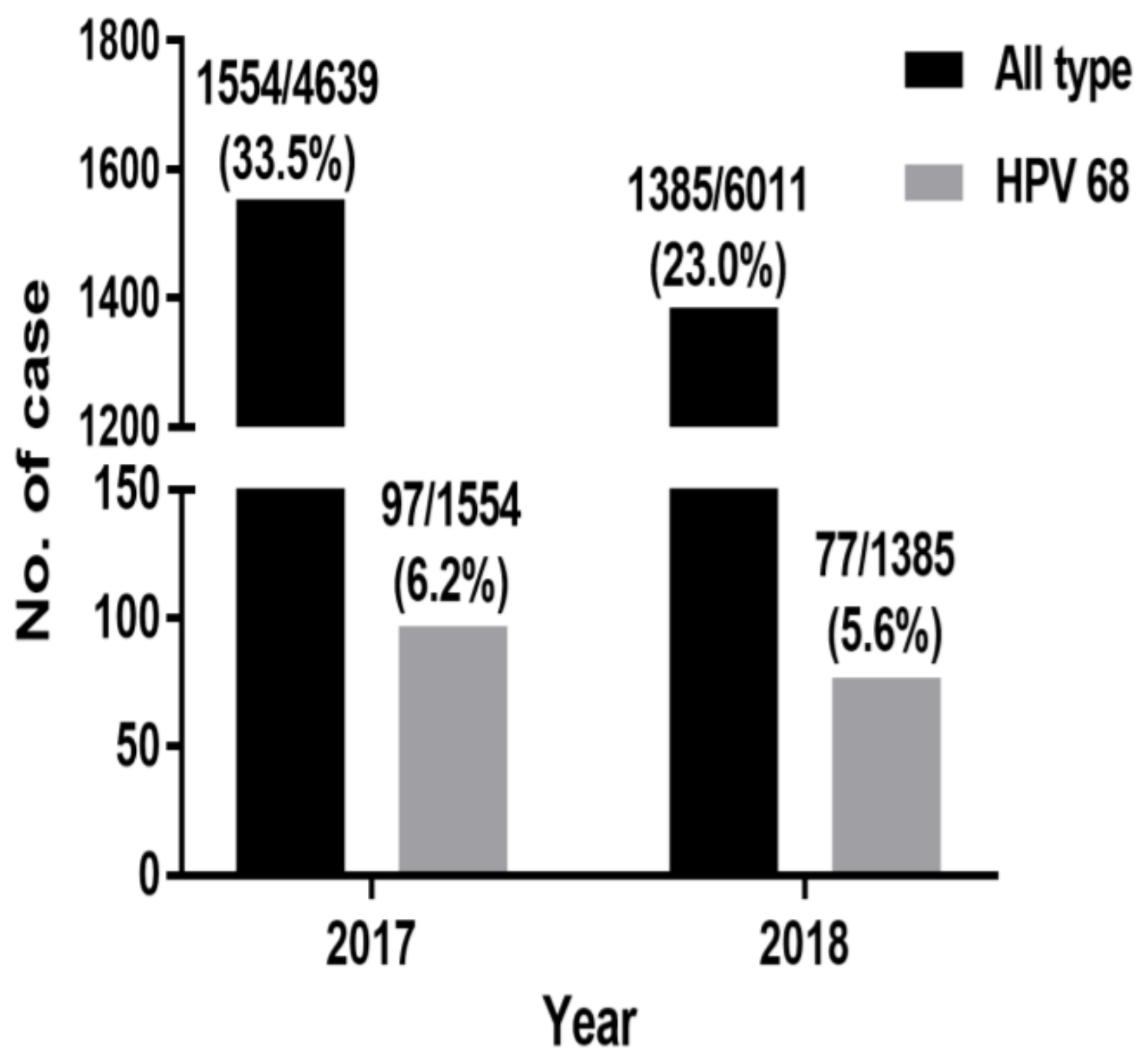

Figure 1 
The number and percentage of HPV68 as well as all type HPV in HPV positive samples during 2017-2018.

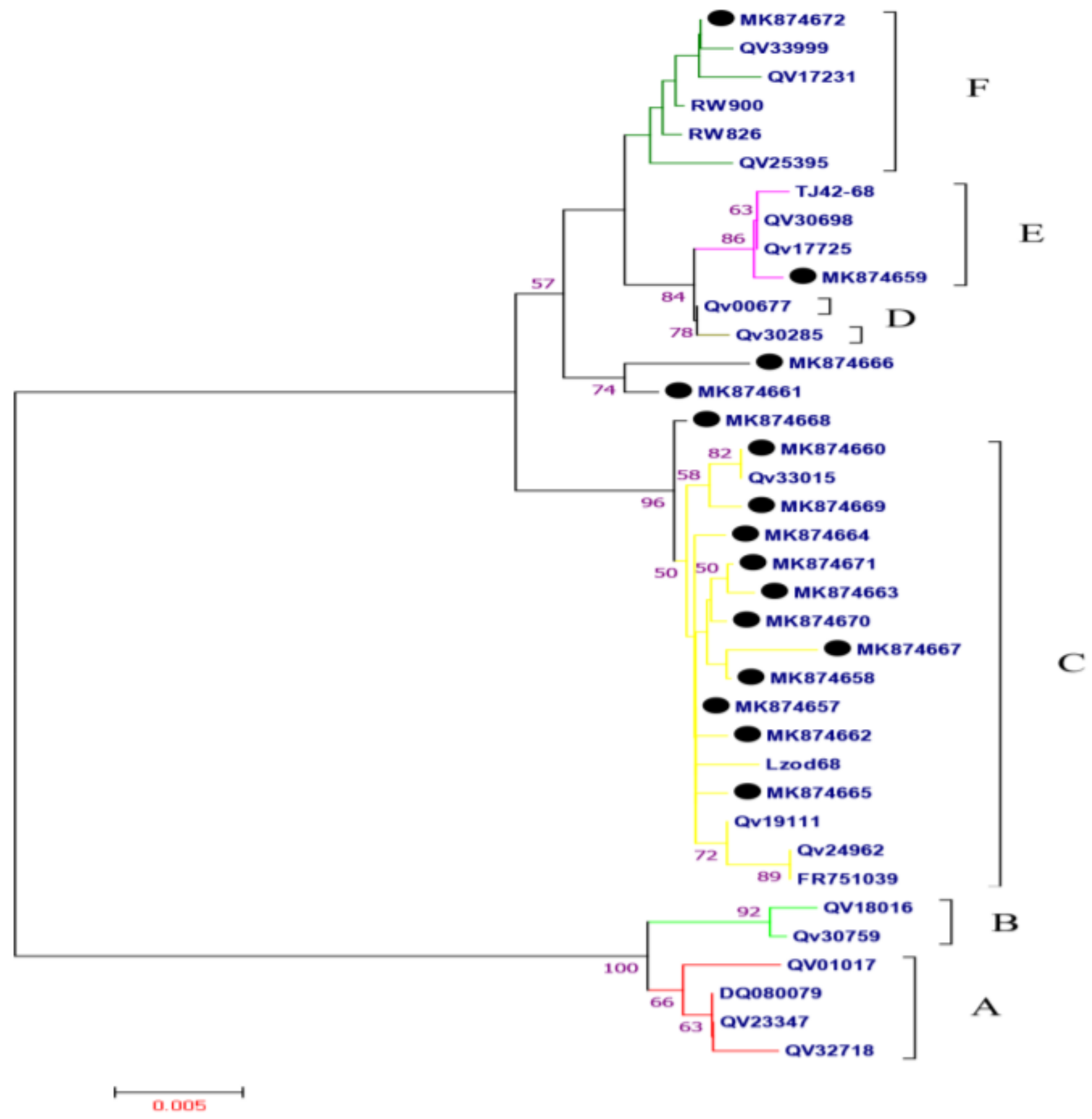

Figure 2

Maximum-likelihood tree of HPV68 variants based on E6-E7 combined sequences. Note: Strains without black dots are reference strains. The reference sequences of lineages are as follows: QV32718, QV23347, DQ80079, QV01017 for A; QV30759, QV18016 for B; FR751039, QV24962, QV19111, Lzod68, QV33015 for C; QV30285, QV00677 for D; QV17725, QV30698, TJ42 for E; QV25395, RW826, RW900, QV17231, QV33999 for F. 
1 MALFHNPEERPYKLPDLCRTLDTTLHDVTI DCVYCRRQL QRTE VYEFAFG 50

51 DLLNVV Y R D G V P L A A C Q S C I K F Y A K I RE L R Y Y S E S V Y A T T L E T I T N T K L Y D 100

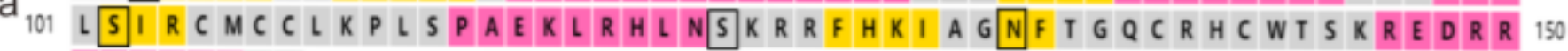
151 R T R Q E T Q V

$\begin{array}{lllll}10 & 20 & 30 & 40 & 50 \\ 10 & 20 & 30 & 40 & 50\end{array}$

1 M A L F H N P E E R P Y K L P D L C R T L D T T L H D V T I D C V Y C R R Q L Q R T E V Y E F A F G 50

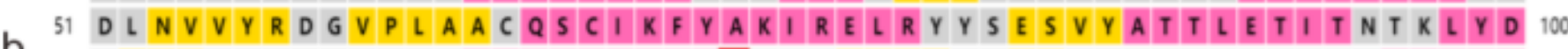

D 101 L S I R C M C C L K P L S P A E K L R H L N A K R R F H K I A G N F T G Q C R H C W T S K R E D R R 150 \begin{tabular}{ll|l|l|l|l|l}
151 & R & $T$ & $R$ & $Q$ & $E$ & T Q V
\end{tabular}

$\begin{array}{lllll}10 & 20 & 30 & 40 & 50 \\ 10 & 20 & 30 & 40 & 50\end{array}$

1 M A L F H N P E E R P Y K L P D L C R T L D T T L H D V T I D C V V Y C R R Q L Q R T E V Y E F A F G 50

C 51 D L N V V Y R D G V P L A A C Q S S C I K K F Y Y A K I R E L R R Y Y S E E S V Y Y A T 101 L S I R C M C C L K P L S P A E K L R H L N S K R R F H K I A G T F T G Q C R H C W T S K R E D R R 150 151 R $T$ T R Q Q E

\begin{tabular}{|c|c|c|}
\hline 10 & 20 & 30 \\
\hline 10 & 20 & 30 \\
\hline
\end{tabular}

1 M A L F H N P E E R P Y K L P D L C R T L D T T L H D V T I D C V Y C R R Q L Q R T E V Y E F A F G 50

d 51 D L H V V Y R D G V P L A A C Q S C I K F Y A K I R E L R Y Y S E S S V Y A T T T L E E I I T N T K L Y D 100 101 L S I R C M C C L K P L S P A E K L R H L N A K R R F H K I A G N F T G Q CR H C W T S K R E D R R 150

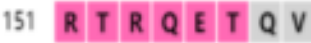

\begin{tabular}{|c|c|c|c|c|}
\hline 10 & 20 & 30 & 40 & 50 \\
\hline 10 & 20 & 30 & 40 & 50 \\
\hline
\end{tabular}

1 M A L F H N P E E R P Y K L P D L C R T L D T T L H D V T I D C V Y C R R Q L Q R T E V Y E F A F G 50

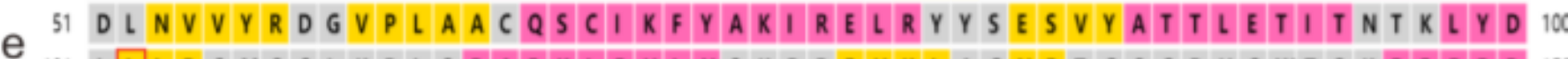
101 L L I R C M C C L K P L S P A E K L R H L N S KRR F H K I A G N F T G Q C R H C WTS KR E D R R 150 \begin{tabular}{lll|l|l|l|l}
151 & R & $T$ & $R$ & $Q$ & $E$ & $T$
\end{tabular}

10 Strand
$\square$ Disordered, protein binding Extracellular

Helix
Putative Domain Boundary
Re-entrant Helix

Coil

Membrane Interaction

Cytoplasmic
40

$\square$ Disordered

Transmembrane Helix

Signal Peptide

\section{Figure 3}

Secondary structure prediction of HPV68 E6 reference and variant sequence. Note: a means HPV68 E6 reference sequence, b means HPV68-MK874661, c means HPV68MK874663, d means HPV68MK874666, e means HPV68MK874669. Amino acids changed are marked with red boxes, and the corresponding amino acids in the reference sequence are marked with black boxes. 
1. MHGPKPTVQEI VIELCPCNEIEPVDLVCHEQLGOSDOEI OEPDHAVNHHQ S

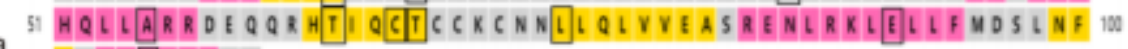

a 10 V CPWEATETQ

$\begin{array}{llll}10 & 30 & 40 & 50 \\ 10 & 20 & 30 & 50\end{array}$

I MHGPKPTVQEIVLELCPCNEI EPVOLVCHEQLGDSDOEI OEPDHAVNHHQ SO

5) HQLIARRDEQQRHTIQCTCCXCNNLLQLVVEASRENLRKLQLIFNOSLNF 100

b 1 V V P WCATETQ

\begin{tabular}{|c|c|c|c|}
\hline 10 & $x$ & 30 & 40 \\
\hline th & $x$ & $y$ & 4 \\
\hline
\end{tabular}

1 MHGPKPTVQEIVLELCPCNEIEPVOLVGHEQLGOSODEI OEPOHAVNHHQSO

51 HQLLARRDEQQRHTIQGTCCKCNNLLQLVVEASRENLRKLQLIFMDSLNF 100

C 101 V P P W ATETQ

$\begin{array}{llll}10 & 30 & 40 & 50 \\ 10 & 20 & 30 & 40\end{array}$

1 MHGPKPTVQEIVLELCPCNEIEPVOLVCHEQLGOSDDEIOEPOHAYNHWQ ST

d 51 HQLIARROEQQRHRIQGVCCKCNNPLQLVVEASRENLRXLQLLF MDSLNF 100

WI VPUCATETQ

$\begin{array}{llll}20 & 30 & 45 & 50 \\ 10 & 30 & 40 & 50\end{array}$

1 MHGP KPTVQEI VLELCPYNEI EPVDLVCHEQLGOSODEI DEPOHAVNHHQ 50

51 HQLLARAOEQQRHTIQCTCCKCNNLLQLYVEASRENLRXLQLIFMDSLNF 100

e vi VACATTQ

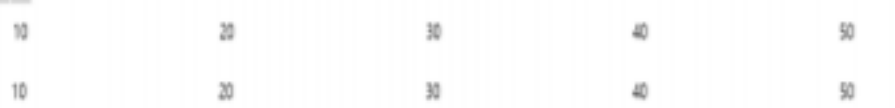

1 MHGPKPTVQEIVLKLCPCNEIEPVOLVCHEQLGOSDOEI OEPOHAVNHHQ SO

5 HQLLARRDEQQRHTIQGTCCXCNNLLQLVVEASRENLRXIQLIFNDSLNF 100

IN VCPWGATETQ

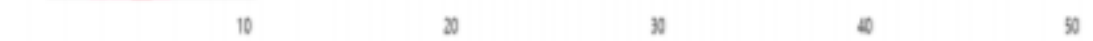

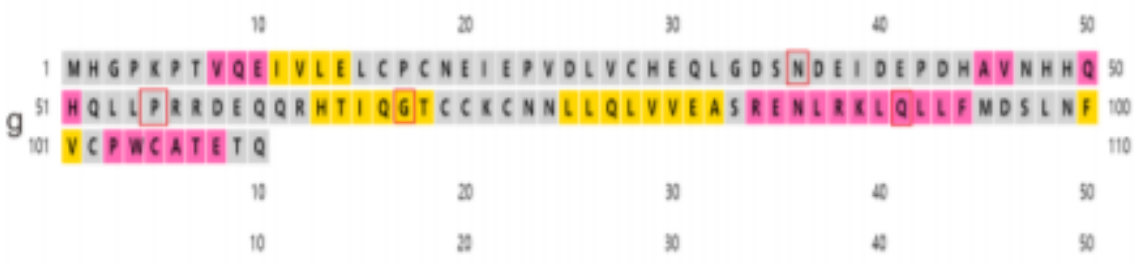

1 MHGPKPTVQEIVLELCPCNEI EPVOLVCHEQLGOSOOEIOEPOHAVNHHQ

h 5 HQLLARRDEQQRHRIQCMCCKCNNDLQLVVEASRENLRXLQLLFNDSLNF 100

h VCPWCATETQ

$10 \quad 30 \quad 30 \quad 50$

\begin{tabular}{|c|c|c|c|}
\hline Strand & Kelin & Coil & Disordened \\
\hline Disordered, protein tinding & Putative Domain Bcundary & Membrane interaction & Transmembrane Helix \\
\hline Entracellatar & Re-entrant Helis & Cytoplasmik & Signal Peptide \\
\hline
\end{tabular}

\section{Figure 4}

Secondary structure prediction of HPV68 E7 reference and variant sequence. Note: a means HPV68 E7 reference sequence, b means HPV68 E7 MK874657, c means HPV68 E7 MK874658, d means HPV68 E7 MK874659, e means HPV68 E7 MK874660, f means HPV68 E7 MK874666, g means HPV68 E7 MK874667, h means HPV68 E7 MK874672. Amino acids changed are marked with red boxes, and the corresponding amino acids in the reference sequence are marked with black boxes. 


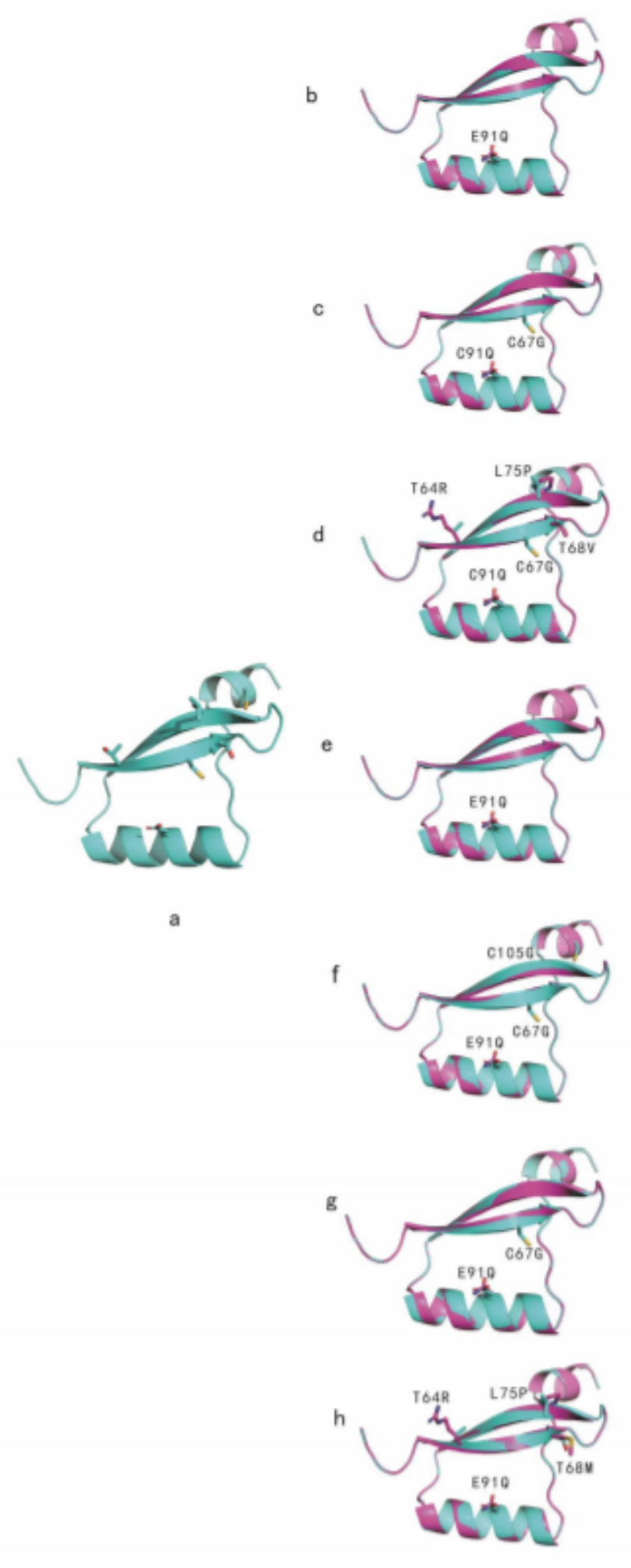

\section{Figure 6}

Tertiary structure prediction of HPV68 E7 reference and variant sequence. Note: Due to template limitations, the tertiary structure of HPV68 E7 58-109 residues were predicted. a means the tertiary structure of HPV68 E7 reference, b means the tertiary structure superposition HPV68 E7 reference and MK874657 sequence, $c$ means the tertiary structure superposition HPV68 E7 reference and MK874658 sequence, $d$ means the tertiary structure superposition HPV68 E7 reference and MK874659 sequence, e 
means the tertiary structure superposition HPV68 E7 reference and MK874660 sequence, $f$ means the tertiary structure superposition HPV68 E7 reference and MK874666 sequence, g means the tertiary structure superposition HPV68 E7 reference and MK874667 sequence, h means the tertiary structure superposition HPV68 E7 reference and MK874672 sequence.

\section{Supplementary Files}

This is a list of supplementary files associated with this preprint. Click to download.

- manscriptforVIROLOGYJOURNALtable.pdf

- SupplementaryMaterialsforVIROLOGYJOURNAL.pdf 